\title{
TRATAMENTO IMUNOSSUPRESSOR NA ESCLEROSE MÚLTIPLA
}

\author{
T. V. OLIVEIRA *-A.M. GORZ-REINHARDT*-C.M. GRACIA * \\ P. R. M. BITTENCOURT**
}

RESUMO - Um estudo aberto, retrospectivo e não-controlado, foi realizado para avaliar a eficácia e tolerabilidade do tratamento imunossupressor instituído no período de 1982 a 1990 em pacientes com diagnóstico de esclerose múltipla clinicamente definida. Dos 42 pacientes, 18 (43\%) foram tratados com uma só droga e 24 (57\%) com mais de uma. A tolerabiliciade foi avaiiada após 6 meses de tratamento em 42 pacientes, dos quais 6 receberam tratamento com prednisona, 26 com azatioprina, 17 com metilprednisolona e 15 com pulsos de ciclofosfamida. Ocorreram efeitos colaterais reversíveis, de intensidade leve a moderada, em 54\% dos pacientes em uso de azatioprina, sendo necessário diminuir a dose ou suspender definitivamente a medicação. Foram observados efeitos adversos em $40 \%$ dos pacientes em uso de pulsos de ciclofosfamida e em 12\% dos pacientes em tratamento com pulsos de metilprednisolona. Efeitos colaterais relacionados ao uso crônico dos corticóides ocorreram em $83 \%$ dos pacientes. Ava¡iaçảo ảa eficácia após 2 anos de tratamento imunossupressor, em 15 pacientes com seguimento adequacio, mostrou aparente parada do progresso da doença, sugerindo benefício da imunossupressão em casos com evolução rápida e inabilidade crescente.

PALAVRAS-CHAVE: esclerose múltipla, terapêutica, drogas imunossupressoras.

Immunesuppressive treatment in multiple sclerosis

SUMMARY - An open, retrospective, uncontrolled study was carried out to evaluate efficacy and tolerability of immunesuppressive therapy used in the period 1982-1990 in patients with clinically definite multiple sclerosis. Of 42 patients $43 \%$ were treated with one drug, and ths others with more than one. Tolerability was evaluated after at least 6 months of therapy in 6 patients on prednisone, 26 on azathioprine, 17 on methyl-prednisolone and 15 on cyclophosphamide pulses. Reversible mild or mocienate side-effects were noted in $54 \%$ of the patients on azathioprine, seading to change in dose or withdrawal. There were similar side-effects in $83 \%$ of the patients on prednisone, $40 \%$ of those on cyclophosphamide, and in $12 \%$ of those on methylprednisoione. Efficacy was evaluated in 15 patients with a follow-up of at least 2 years, measured by objective scales. The results indicated therapy appeared to arrest progress of disability. We conclude that this study supports the use of immunesuppresssion in selected cases with rapidly evolving neurologizal deficits.

KEY WORDS: multipie sclerosis, therapeutics, immunesuppressive drugs.

Estudos quanto à patogênese da esclerose múltipla (EM) sugerem um me canismo auto-imune, relacionado a uma infecção viral, em pessoas geneticamente predispostas. Modalidades diferentes de tratamento, visando a suprimir ou restaurar o sistema imune, têm sido utilizadas com resultados controversos 1-5,9,10,14, 15,17-19. A história natural pode variar desde uma evolução fulminante, levando a óbito dentro de algumas semanas do início, até doença completamente assinto mática descoberta incidentalmente na necrópsia. Três tipos de evolução são bem

Unidade de Neurologia Clínica dos Hospitais Nossa Senhora das Graças e Santa Cruz, Centro Diagnóstico de Curitiba e Centro Dia - Clínica de Recuperação, Curitiba: *Neurologista; **Neurologista, Chefe da Unidade.

Dra. Teresinha Valente de Oliveira - Unidade de Neurologia Clínica, Hospital Nossa Senhora das Gracas - Rua Alcides Munhoz 433 - 80510 Curitiba PR - Brasil. 
determinados: recidivas intercaladas com remissões; progressão contínua após período variável de remissões e recidivas; e progressão crônica com deterioração contínua sem remissão, desde o início 20. Tentativas para modificar o curso da EM têm sido realizadas com drogras imunossupressoras de várias formas. Há aproximadamente três décadas são utilizados corticóides nas crises de desmielinização 18. Este tratamento foi aprimorado com a utilização de pulsos de metilprednisolona, que parecem ter efeito imunossupressor pelo menos discreto 3,9 Corticóides utilizados cronicamente são medida de última instância, tendo em vista os efeitos colaterais 18 . Imunossupressão crônica tem sido obtida com ciclosporina e azatioprina administrados por via oral diariamente $14,15,17$ ou por pulsoterapia com ciclofosfamida administrada endovenosamente a intervalos de $1-3$ meses 4,5,10,16,19.

Neste estudo retrospectivo e aberto revisamos o tratamento imunossupressor de pacientes com EM, com a finalidade de avaliar, de maneira descritiva, a tolerabilidade e a eficácia destas drogas utilizadas no sistema médico brasileiro.

\section{CASUISTICA E METODOS}

Revisamos os prontuários de todos os pacientes atendidos na Unidade de Neurologia Clínica (Hospital Nossa Senhora das Graças, Hospital Santa Cruz, Centro-Dia e Centro Diagnóstico de Curitiba), no período de 1982 a outubro-1990, com diagnóstico definido, prováve e possivel de EMī. Foram selecionados os pacientes com diagnóstico de EM clinicamente definida, de acordo com os critérios de Poser et al 11,13. Estes são pacientes com: história clinica compatível, com pelo menos dols episódios de desmielinização e manifestações clínicas de duas lesões separadas no sistema nervoso central; ou aqueles com um episódio de desmielinização associado la uma evidência clínica e outra paraclínica de lesão separada. Como métodos paraclínicos, são aceitos: potencial evocado sômato-sensitivo, visual e/ou auditivo; imagem por ressonância magnética e/ou tomografia computadorizada de crânio. Excluímos deste estudo os casos classificados como EM provável e possível 11 .

Todos os pacientes tiveram suas histórias clínica e terapêutica revisadas em detailhe sempre que nouvessem dado específicos, isto é, drogas, doses, tempo de tratamento e resposta clinica, mesmo que condutas terapêuticas tivessem sido tomadas anteriormente em outros serviços. A doença foi classificada, de acordo com a evolução, em três formas: remissão e recidivas, exlacerbação com progressão, e progressiva desde o início. O levantamento dos dados Ioi retrospectivo.

Foram considerados para a avaliacão da tolerabilidade todos os pacientes com pelo menos 6 meses de seguimento, em tratamento com uma ou mais drogas imunossupressoras. Só foram considerados pacientes que tivessem seguido uma das rotinas de tratamento padronizadas na Unidade de Neurologia Clínica como imunossupressoras, em termos gerais, não especificamente no que consideramos indicado em EM. Estas são prednisona via oral (VO) (60-80mg/dias alternados), azatioprina vo $(2,5 \mathrm{mg} / \mathrm{kg} / \mathrm{dia})$, pulsos de metilprednisolona endovenosa (EV) (1 $\mathrm{g} / \mathrm{dia}$ por 3 ou 5 dias) $\delta$ e pulsos de cidiofosfamida $E V(750 \mathrm{mg} / \mathrm{m} 2$ de superfície corporal) 5 . A tolerabilidade foi avaliada de acordo com o tipo e dose da drogia, o tempo de uso, o tipo e intensidade de efeito colaterial, se reversivel ou não, se houve necessidade de suspender ou diminuir a cose para controlar o efeito aaverso e se ocorreu sequela em função da medicação. A eficácia foi medida nos pacientes com pelo menos dois anos de seguimento clínico periódico, no qual os dados de história e exame físico pudessem ser computados e aplicados nu escala de inabilidade de Kurtzke 6,7. A apresentação dos resultados foi feita de maneira descritiva, devido à natureza não controlada e retrospectiva do estudo.

\section{RESULTADOS}

A idade de início dos sintomas e do tratamento, assim como a do final do seguimento encontram-se na Tabeia 1. Havia 33 pacientes do sexo feminino e 9 do masculino. Dos 42 pacientes, em 3 ocorreu o óbito, 2 em decorrência de curso de exacerbação, com rápida progressão da doença, e 1 com evolução progressiva, com óbito não relacionado. Havia 27 pacientes (64\%) evoluindo sob a forma de recidiva e remissão, 12 pacientes (33\%) com recidivas seguidas de progressão e 3 pacientes (7\%) com progressão desde o início.

O tratamento foi realizado com uma droga em 18 (43\%) pacientes e com associação de duas ou mais em 24 (57\%). Na Tabela 2 encontra-se a relação dos tratiamentos isolados instituídos. 
A tolerabilidade ao uso de prednisonia por pelo menos 6 meses foi avaliada em 6 pacientes. O tempo médio de uso foi 29 meses (6-60 meses, variacão), com dose média de $73 \mathrm{mg}$ de prednisona em dias alternados. Cinco pacientes (83\%) apresentaram efeitos adversos pelo uso crônico, como síndrome de Cushing (4), aủteração mental (2) e hipertensão arterial (1). Estes efeitos colaterais foram de intensidade moderada e reversiveis com a suspensão da medicação.

Tabela 1. Idade de início dos sintomas de esclerose múltipla (EM) ( $n=42$ ), idade ao serem submetıdos pela primeira vez a tratamento imunossupressor de $\mathbf{E M}(n=42)$ e idade na última avaliação clínica $(n=39)$.

\begin{tabular}{ccc}
\hline & $\begin{array}{c}\text { Média } \\
\pm M\end{array}$ & $\begin{array}{c}\text { Mínimo-máximo } \\
\text { desvio padrão }\end{array}$ \\
\hline \hline Idade de início (anos) & $33 \pm 10,5$ & $16-55$ \\
Idade no início do tratamento (anos) & $37 \pm 9,9$ & $17-55$ \\
Idade no fim do seguimento (anos) & $41 \pm 9,2$ & $26-59$ \\
\hline
\end{tabular}

Tabela 2. Número e natureza dos esquemas terapêticos instituidos em 42 pacientes com Eır.

\begin{tabular}{|c|c|c|c|c|}
\hline Medicações & $\begin{array}{c}\text { No de } \\
\text { tratamentos }\end{array}$ & $\%$ & Monoterapia & Politerapia \\
\hline Prednisona & 6 & 9 & 0 & 6 \\
\hline \multirow[t]{2}{*}{ Metilprednisolona } & 12 & 19 & 4 & 8 \\
\hline & 5 & 8 & 2 & 3 \\
\hline Azatioprina & 26 & 41 & 12 & 14 \\
\hline Ciclofosfamida & 15 & 23 & 7 & 8 \\
\hline Total & 64 & 100 & 25 & 39 \\
\hline
\end{tabular}

A pulsoterapia com metilprednisolona foi prescrita num total de 17 ciclos, em 17 pacientes: em 12 de 3 dias e em 5 de 5 dias. Efeitos colaterais agudos ocorreram em 2 (12\%) pacientes, respectivamente micose superficial de pele e candidíase oral. Ambas as infeccōes foram de curta duração, leves em intensidade e reversíveis com medicação antifúngica.

A tolerabilidade da azatioprina foi avlaliada em 26 pacientes após tempo médio de tratamento de 35 meses, variando a partir de poucos dias, com a interrupção da medicação por intolerância gastrointestinal em dois pacientes, até 72 meses, após a suspensão por uso rrolongado em $6(23 \%)$ pacientes. A interrupção ocorreu após tempo médio de 50 meses de tratamento, apesar da boa tolerabilidade. Efeitos adversos, de intensidade moderada e reversiveis com a suspensão da medicação, ocorreram em 7 (27\%) pacientes. Em outros 7 (27\%) pacientes, foi necessário diminuir a dose da azatioprina devido a efeitos leves e reversíveis (Tabela 3 ).

Infecção na vigência da azatioprina e provavelmente relacionada a seu uso ocorreu em apenias 1 (4\%) dos pacientes, que apresentou herpes zoster e neuropatia pós-herpética no membro superior esquerdo. Houve infecções na vigência de azatioprina, talvez relacionadas com doença, mas não com a droga específica: infecção urinária ocorreu em 4 (15\%) pacientes, broncopneumonia em $1(4 \%)$ e amigdalite em 1 (4\%) dos pacientes.

A pulsoterapia com ciclofosfamida foi prescrita em 15 pacientes, num total de 42 ciclos, variando de 1 a 9 ciclos por paciente. Dez pacientes utilizaram a droga por menos de 6 meses. Foi observada intolerância gastrointestinal após a infusão em 3 (20\%) dos pacientes 
Tabela 3. Efeitos colaterais observados em $14(54 \%)$ de 26 pacientes tratados com azatioprina. Em 7 deles (27\%) foi necessária diminuição da dose e em 7 (27\%) a droga foi suspensa.

\begin{tabular}{|c|c|c|}
\hline & No de pacientes & $\%$ \\
\hline Leucopenia leve & 6 & 23 \\
\hline Intolerância gastrointestinal & 2 & 8 \\
\hline Leucopenia moderada & 2 & 8 \\
\hline Pancitopenia moderada & 1 & 4 \\
\hline Elevação moderada das transaminases séricas & 1 & 4 \\
\hline Lesões de pele (arterite) & 1 & 4 \\
\hline Elevação leve das transaminases & 1 & 4 \\
\hline
\end{tabular}

e alopécia moderada e reversível em 1 (7\%). Cinco pacientes utilizaram a droga por mais de 6 meses, com média de tempo de tratamento de 20 (10 a 31 meses de variação) meses e média de 7 ciclos de tratamento por paciente. Em 2 (13\%) dos pacientes ocorreu quadro clínico de menopausa precoce. Em uma paciente foi necessário suspender a medicação sem melhora dos sintomas. Em outra foi reduzida a dose de ciclofosfamida para a metade, com melhora dos sintomas (Tabela 4).

Tabela 4. Efeitos colaterais observados em 6 de 15 pacientes (40\%) com EM tratados com pulsos de ciclofosfamida.

\begin{tabular}{lcc}
\hline & No de pacientes & $\%$ \\
Intolerância gástrica (náuseas, vômitos) \\
Menopausa precoce \\
Alopécia leve
\end{tabular}

A eficácia do tratamento, em 15 pacientes em uso de uma ou mais drogas por no minimo 2 anos, foi avaliada pela aplicação da escala de estado de inabilidade 6 no início do tratamento e ao final da avialiação clínica (Tabela 5). Após dois anos de tratamento imunossupressor, houve piora clínica em 9 pacientes; em três o quadro neurológico permaneceu inalterado; outros três obtiveram melhora clínica. Em um destes a melhora foi clinicamente significante: total inabilidade física com restrição ao leito evoluiu para mínima inabildade; o pacente chegou a locomover-se sem apoio e tornou-se independente.

A variação na escala do estado de inabilidade 6 de 4,7 para 5,1 , em termos práticos, significa que o grau de deficiência neurológica progrediu, no grupo como um todo, resultando em maior inabilidade física.

\section{COMENTARIOS}

Está bem definido por vários estudos que os corticóides são eficazes na fase aguda da EM, podendo induzir remissão. Têm sido utilizados com frequência esquemas com altas doses por curto período de tempo, impedindo os efeitos indesejáveis do uso prolongado $3,8,9,18$.

Num estudo duplo-cego utilizando altas doses de metilprednisolona endovenosa foi observado efeito terapêutico significativo, sem efeitos adversos sérios. 
Tabela 5. Eficácia do tratamento imunossupresor em 15 pacientes com EM após 2 anos de tratamento com uma ou mais drogas e sua correlação a mudanģas na escala do estado de inabilidade entre o início do tratamento e o final do período de observação.

\begin{tabular}{|c|c|c|c|c|c|c|c|c|}
\hline $\begin{array}{l}\text { No do } \\
\text { paciente }\end{array}$ & Droga-1 & $\begin{array}{l}\text { Tempo de } \\
\text { tratamento } \\
\text { (meses) }\end{array}$ & Droga-2 & $\begin{array}{c}\text { Tempo de } \\
\text { tratamento } \\
\text { (meses) }\end{array}$ & Droga-3 & $\begin{array}{c}\text { Tempo de } \\
\text { tratamento } \\
\text { (meses) }\end{array}$ & $\begin{array}{c}\text { DSS } \\
\text { inicial }\end{array}$ & $\begin{array}{r}\text { DSS } \\
\text { final }\end{array}$ \\
\hline 1 & AZA & 72 & & & & & 7 & 8 \\
\hline 2 & $A Z A$ & 36 & & & & & 6 & 6 \\
\hline 3 & AZA & 48 & & & & & 5 & 7 \\
\hline 4 & AZA & 29 & & & & & 4 & 6 \\
\hline 5 & AZA & 30 & & & & & 5 & 3 \\
\hline 6 & AZA & 46 & & & & & 4 & 3 \\
\hline 7 & $\mathrm{AZA}$ & 43 & & & & & 3 & 6 \\
\hline 8 & $\mathbf{A Z A}$ & 39 & & & & & 5 & 5 \\
\hline 9 & AZA & 36 & & & & & 2 & 3 \\
\hline 10 & AZA & 60 & $\mathbf{P}$ & 36 & & & 9 & 2 \\
\hline 11 & AZA & 65 & $\mathbf{P}$ & 41 & & & 6 & 7 \\
\hline 12 & $\mathbf{A Z A}$ & 39 & $\mathbf{P}$ & 6 & & & 2 & 2 \\
\hline 13 & AZA & 66 & $\mathbf{P}$ & 60 & CFM & 19 & 4 & 6 \\
\hline 14 & AZA & 44 & & & CFM & 22 & 5 & 8 \\
\hline 15 & AZA & 48 & & & CFM & 31 & 4 & 5 \\
\hline Média & - & 47 & - & 36 & - & 24 & 4,7 & 5,1 \\
\hline
\end{tabular}

$A Z A$, azatioprina; $P$, prednisona; CFM, aiclofosfamida; DSS, disability status scale (escala do estado de inabilidade).

Os efeitos adversos mais frequentemente registrados foram leve rubor de face, edema transitório de tornozelo e gosto metálico na boca durante a infusão. Estes sintomas foram leves e nunca tão severos quanto os observados durante cursos prolongados de ACTH intramuscular ou corticóide oral ${ }^{9}$. Em estudo com 350 cursos de tratamento com altas doses de metilprednisolona em recidivas da EM foi observado pequeno número de efeitos adversos transitórios, como infecções menores, infecção de trato urinário baixo, candidíase oral e vaginal; não ocorreram infecções sistêmicas ou disseminadas; as queixas gastrointestinais foram infrequentes; ocorreram hiperglicemia leve e complicações psiquiátricas 8 . Pulsoterapia com metilprednisolona vem sendo utilizada em nossa Unidade nas recidivas de EM desde 1988, com poucos efeitos indesejáveis. As complicações de corticóide via oral, em altas doses e por tempo prolongado, estāo sendo evitadas.

A utilização da azatioprina em nossa Unidade foi iniciada em 1983, quando somente alguns estudos preliminares indicavam benefícios no tratamento da EM, diminuindo a frequência das exacerbações. Encontramos, como relatado na literatura 15, alterações transitórias nos exames laboratoriais, como discrasias sanguíneas e elevação das transaminases. Estas anormalidades foram sempre reversíveis com a diminuição ou, mais raramente, suspensão da droga. Não encontramos infecções sistêmicas severas que levassem a risco de vida. Até o momento nenhum paciente desenvolveu neoplasia, apesar do uso prolongado. Em virtude de haver estudos recentes relatando tumores sólidos após 1,4 a 2,7 anos de tratamento com azatioprina 1 , passamos a sugerir a suspensão da azatioprina nos pacientes com mais de 5 anos de tratamento.

A administração diária de ciclofosfamida via oral tem o riso de complicações urinárias, infecção e anomalias cromossômicas que podem levar a linfoma e leucemia 12. Outros efeitos colaterais da ciclofosfamida em uso contínuo são carcinoma secundário, leucopenia severa, cistite hemorrágica, alopécia, amenor réia, fadiga, herpes simples, infecção bacteriana, piúria asséptica, hematúria mi- 
croscópica ou grosseira, úlcera da mucosa vesical, anemia leve, discreta elevação da fosfatase alcalina, náuseas, vômitos e urticária 5,10. A maioria dos estudos tem sido em pacientes com EM progressiva 4,10. Em um destes, com seguimento de 2 anos, foi utilizado esquema de indução com altas doses de ciclofosfamida via endovenosa, seguido por terapia áe manutenção com pulsoterapia mersal. Foram observados efeitos tóxicos agudos, como náuseas, vômitos e alopécia completa. Estes obstáculos importantes ao tratamento são associados aos riscos crônicos do uso de ciclofosfamida, como possível relação causal com carcinoma de bexiga, leucemia e potencial mutagênico nas gerações futuras 4 .

A administração de ciclofosfamida por pulsos, por via endovenosa, com intervalos de várias semanas, tem demonstrado reduzir a frequência de exacerbações. O tratamento intermitente limita o risco das complicaçōes. O risco da irritação de bexiga pode ser evitado com administração endovenosa de grandes doses de líquidos nas 24 horas antes e após o tratamento. A administração profilática de antieméticos reduz as náuseas e a monitorização do sangue, com a contagem de leucócitos, reduz o risco de leucopenia severa 16. Efeitos indesejáveis agudos e a curto prazo, observados em nossos pacientes a partir de 1988 quando iniciamos tratamento com pulsos de ciclofosfamida, são já frequentemente relatadoś na literatura. Em nosso restrito grupo de pacientes, estas medidas posiribilitaram o uso prolongado de ciclofosfamida, atingindo média de 20 meses. $O$ único efeito adverso de maiores consequências foi menopausa precoce, o que nos levou a especificar esta possibilidade em todas as novas candidatas ao trat:amento. Deve ser lembrado, no entanto, que os dois casos ocorreram em mulheres de 35 e 40 anos.

Existem aspectos metodológicos deste estudo que devem ser enfatizados. Suas características de amostragem retrospectiva e a ausência de grupo controle impossibilitam qualquer afirmativa definitiva sobre a real eficácia terapêtica dos medicamentos utilizados. Devido às características particulares da medicina brasileira é de esperar que pacientes com maus resultados tenham abandonado o tratamento. Nossa casuística deve incluir desvio importante para resultados positivos, ou seja, somente teriam sido seguidos por 2 anos os pacientes corn sucesso no tratamento. A observação de que praticamente não houve deterioraçăo neurológica nestes casos é importante, servindo de apoio a parte da literatura internacional sobre o assunto, mas não deve ser colocada como evidência indiscriminada da eficácia terapêutica.

Os resultados podem ser interpretados como indicando que um subgrupo importante de pacientes com EM se beneficia de tratamento imunossupressor. Este subgrupo parece estar localizado na porção mais maligna do espectro de pacientes com EM. Nestes casos, o tratamento imunossupressor pode parar a evolução da deterioração neurológica, embora com algum custo em morbidade pela imunossupressão crônica. Os pacientes apresentaram, em geral, boa tolerabilidade e os efeitos colaterais foram em pequeno número e reversíveis. Nenhum apresentou efeitos colaterais graves, como o desenvolvimento de neoplasia.

Existem outras formas de tratamento de EM, também dependentes de imunossupressão. A utilização continuada de ciclosporina parece retardar a evolução da doença e alterar para melhor o grau nas escalas de atividade funcional. Efeitos colaterais com o uso prolongado, tais como toxicidade renal, hipertensão arterial, distúrbios gastrointestinais, hipertricose, cefaléia e anemia, são relatados 15,17. Outros estudos têm tentado, ainda sem reconhecimento internacional, demonstrar efeito benéfico de COP 1 em pacientes com a forma de exacerbação e remissão.

Nossos resultados parecem indicar a possibilidade da utilização de tratamento imunossupressor em centros com experiência e estrutura para o acompanhamento das intercorrências. Nossa recomendação atual é de pulsos de metilprednisolona nas recidivas de desmielinização; nos quadros de deterioração ne ırológica progressiva secundária, ou recidivas frequentes, sugerimos a associação de pulsos de metilprednisolona e ciclofosfamida. Em casos especiais, como por exemplo durante a gravidez concomitante a atividade da doença, tem sido utilizada a azatioprina. A decisão final sobre a forma de tratamento deve ficar com o paciente, que deve ser informado sobre a evolução natural da doença e das formas de tratamento. 
Agradecimentos - Os autores reconhecem o apoio da comunidade médica do Paraná e Santa Catarina, bem como do Compo Clínico do Hospital Nossa Senhora das Graças, em especial da equipe de Neurocirurgia (Serviço do Prof. Affonso Antoniuk), por referir pacientes para o tratamento imunossupressor. Agradecenos ainda à Marli Osna pela boa-vontade no processamento de palavras.

\section{REFERENCIAS}

1. Amato MF, Siracusa AG, Fratiglioni, L, Amaducci L. Azathioprine therapy and cancer risk in multiple sclerosis: a prospective long-term study. Ann Neurol 1990, 28:255-282.

2. Bornstein $\mathbf{M B}$, Miller A, Slagle $\mathbf{S}$, Weitzman M, Crystal $H$, Drexler E, Keilson $\mathbf{M}$, Merriam A, Wassertheil-Smoller S, Spada V, Weiss W, Arnon R, Jacobsohn I, Teitelbaum $\mathcal{D}$, seia $M$. A pilot trial of cop 1 in exacerbating-remitting multiple sclerosis. $N$ Engl J Med 1987, 317:408-414.

3. Dureli L, Cocito D, Riccio A, Barile C, Bergamasco B, Baggio GF, Perla F, Delsedime M, Gusmaroli G, Bergamini L. High-dose intravenous methylprednisolone in the treatment of multiple sclerosis: clinical-immunologic correlations. Neurology 1986, 36:238-243.

4. Gooukin DE, Plencner S, Palmer-Saxerua J, Teetzen M, Hertsgaard D. Cyciopuosphamiae in chronic progressive multiple sclerosis. lWaintenance vs nonmaintenance therapy. Arch Neurol 1987, 44:823-827.

5. Killian JM, Bressler RB, Armstrong RM, Huston DP. Controlled pilot trial of monthly intravenous cyclophosphamide in multiple sclerosis. Arch Neurol 1988, 45:27-30.

6. Kurtzke JF. Neurological impairment in multiple sclerosis and the disability status scale. Acta Neurol Scand 1970, 46:493-512.

7. Kurtzke JF. A proposal for a uniform minimal record of disability in multiple sdierosis Acta Neurol Scand 1981, 64:110-129.

8. Lyons PR, Newman PK, Saunders M. Methylprednisolone therapy in multiple sclerosis: (a profile of adverse effects. $J$ Neurol Neurosurg Psychiatry 1988, 51:285-287.

9. Milligan NM, Newcombe R, Compston DAS. A double-blind controlled trial of high dose methylprednisolone in patients with multiple sclerosis: clinical effects. $J$ Neurol Neurosurg Psychiatry 1987, 50:511-516.

10. Myers LW, Fahey JL, Moody DJ, Mickey MR, Frane MV, ENison GW. Cyclophospha mide pulses in chronic progressive multiple sclerosis: a preliminary clinical trial. Arch Neurol 1587, 44:828-832.

11. Uliveira TV, Carvalho RM, Seixas RR, Gorz AM, Bittencourt PRM. Esclerose múltipla: critérios objetivos de aiagnóstico. Arq Neuro-psiquiat (São Paulo), 1991, 49:33-42.

12. Fotz PH, Klippel JH, Decker JL, Grauman D, Wolff B, Brown BC, Rutt G. Bladder complications in patients receiving cyclophosphamide lor systemic lupus erythematosus or rneumaioid archritis. Ann Intern Med 19/9, 91:221-223.

13. Poser CM, Paty DW, Scheinberg L, MacDonald WI, Davis FA, Ebers GC, Johnson KP, Sibiey WA, Silberbergh DH, Tourtellotte WW. New diagnostic criteria for multiple sclerosis: guidelines for research protocols. Ann Neurol 1983, 13:227-231.

14. Rudge $\mathfrak{F}$, Koetsier JC, Mertin J, Mispeblom Beyer JO, Vanwalbeek HK, Clifford Jones R, Harrison J, Robinson $K$, Meilein B, Poole $T$ Stokvis JCJM, Timonen P. Kandomised double blind controlled trial of cyclosporin in multiple sclerosis. $J$ Neurol Neurosurg Psychiatry 1989, 52:559-565.

15. Steck AJ, Regli F, Ochsner F, Gauthier G. Cyclosporine versus azathioprine in the treatment of multiple sclerosis: 12-month clinical and immunoiogical evaluation. Eur Neurol 1990, 30:224-228.

16. Steinberg AD. Cyclophosphamide: should it be used daily, monthly, or never? $N$ Engl J Med 1984, 16:458-459.

17. The Multiple Sclerosis Study Group. Efficacy and toxicity of cyclosporine in chronic progressive multiple sclerosis: a randomized, double-blinded, placebo-controlled clinical trial. Ann Neurol 1990, 27:591-605.

18. Troiano R, Cook SD, Dowling PC. Steroid therapy in multiple slcerosis: point of view. Arch Neurol 1987, 44:803-807.

19. Weiner HL, Hafler DA. Immunatherapy of multiple sclerosis. Ann Neurol 1988, 23:211-222.

20. Weinshenker BG, Bass B, Rice GPA, Noseworthy J, Carriere W, Baskerville J, Ebers GC. The natural history of multiple sclerosis: a geographically based study I. Clinical Course and disability. Brain 1989, 112:133-146. 